\title{
Development of a Nomogram Model for Predicting the Risk of In-Hospital Death in Patients with Acute Kidney Injury
}

\author{
Xiuying Yao'** \\ Lixiang Zhang ${ }^{2}$ ** \\ Lei Huang ${ }^{l} * *$ \\ Xia Chen ${ }^{2, *}$ \\ Li Geng' \\ $\mathrm{Xu} \mathrm{Xu}{ }^{1}$
}

'Department of Intensive Care Unit, The First Affiliated Hospital of USTC, Division of Life Sciences and Medicine, University of Science and Technology of China, Hefei, Anhui, 230036, People's Republic of China; ${ }^{2}$ Department of Nursing DepartmeThe First Affiliated Hospital of USTC, Division of Life Sciences and Medicine, University of Science and Technology of China, Hefei, Anhui, 230036, People's Republic of China

*These authors contributed equally to this work
Correspondence: Xiuying Yao

Department of Intensive Care Unit, The First Affiliated Hospital of USTC, Division of Life Sciences and Medicine, University of Science and Technology of China, No. I, Swan Lake Road, Hefei, Anhui Province, People's Republic of China Tel +86-18963782620

Email xiuyingyao@।26.com
Objective: To analyze the risk factors of in-hospital death in patients with acute kidney injury (AKI) in the intensive care unit (ICU), and to develop a personalized risk prediction model. Methods: The clinical data of 137 AKI patients hospitalized in the ICU of Anhui provincial hospital from January 2018 to December 2020 were retrospectively analyzed. Patients were divided into two groups: those that survived to discharge ("survival" group, 100 cases) and those that died while in hospital (“death" group, 37 cases), and risk factors for in-hospital death analyzed.

Results: The in-hospital mortality of AKI patients in the ICU was $27.01 \%(37 / 137)$. A multivariate logistic regression analysis indicated age, mechanical ventilation and vasoactive drugs were significant risk factors for in-hospital death in AKI patients, and a nomogram risk prediction model was developed. The Harrell's C-index of the nomogram model was 0.891 (95\% CI: 0.837-0.945), and the area under the receiver operating characteristic (ROC) curve was 0.886 (95\% CI: $0.823-0.936)$ after internal validation, indicating that the nomogram model had good discrimination. The Hosmer-Lemeshow goodness of fit test and calibration curve indicated the predicted probability of the nomogram model was consistent with the actual frequency of death in ICU patients with AKI. The decision curve analysis (DCA) showed that the clinical net benefit level of the nomogram model is highest when the probability threshold of AKI is between 0.01 and 0.75 .

Conclusion: Patients in the ICU with AKI had high in-hospital mortality and were affected by a variety of risk factors. The nomogram prediction model based on the risk factors of AKI showed good prediction efficiency and clinical applicability, which could help medical staff in the ICU to identify AKI patients with high-risk, allowing early prevention, detection and intervention, and reducing the risk of in-hospital deaths in ICU patients with AKI.

Keywords: AKI, ICU, inpatient mortality, risk factors, prediction risk model

\section{Introduction}

Acute kidney injury (AKI) is one of the most common critical diseases in hospitalized patients and has a high incidence rate and mortality. ${ }^{1}$ Studies $^{2}$ reveal that more than $15 \%$ of hospitalized patients and $60 \%$ of critically ill patients may develop AKI. With age, as body functions gradually decline, and when combined with multiple organ diseases, the incidence rate of AKI gradually increases. The complication of AKI in inpatients often leads to a prolonged hospital stay, creating an increased burden of hospitalization expenses, increased hospital mortality, permanent damage in renal function, etc. The mortality rate of AKI inpatients can be as high as $15.8-22.4 \%{ }^{3,4}$ 
A multicenter survey in China showed that the median length of stay for AKI inpatients in general hospitals in China is 18 days, and the in-hospital mortality rate is $12.4 \%{ }^{5}$ Feng et al, ${ }^{6}$ in a Chinese multicenter prospective epidemiological study of AKI, found that in-hospital mortality rate of AKI inpatients was $9.24 \%$, that nephrology and ICU were high risk departments for AKI, especially critically ill patients in the ICU, and that AKI was an independent risk factor for increased mortality.

AKI not only increases the mortality of patients, but also may lead to the occurrence of chronic kidney disease (CKD), end-stage renal disease (ESRD) and adverse cardiovascular events. ${ }^{7}$ Therefore, it is clinically important to identify the relevant risk factors of a short-term adverse prognosis from AKI and take targeted intervention strategies to reduce the risk of such events. This study retrospectively analyzed the clinical data of 137 AKI patients hospitalized in the ICU of Anhui provincial hospital from January 2018 to December 2020 and developed the first nomogram prediction model to predict the risk of inhospital death from AKI in China. The goal is to screen AKI patients for those with a high risk of death so that targeted prevention and intervention strategies can be taken to reduce the incidence of in-hospital death in AKI patients.

\section{Materials and Methods Research Subjects}

Research subjects were 137 AKI patients hospitalized in the ICU of Anhui provincial hospital from January 2018 to December 2020. The inclusion criteria were as follows. 1) All research subjects met the AKI diagnostic criteria recommended by KDIGO guidelines, ${ }^{8}$ ie, they met one of the following three conditions: an increased serum creatinine (SCr) level $\geq 26.5 \mu \mathrm{mol} / 1$ within $48 \mathrm{~h}$; a SCr level within 7 days that was 1.5 times the baseline level; or, a urine volume $<0.5 \mathrm{~mL} / \mathrm{kg} / \mathrm{h}$ for more than $6 \mathrm{~h}$. 2) Clinical data were complete. 3) Length of hospital stay was $>24 \mathrm{~h}$. Exclusion criteria were: 1) baseline SCr level $<40 \mu \mathrm{mol} / \mathrm{L}$; and 2) patients with a kidney transplant or on maintenance hemodialysis. The study protocol was approved by the medical ethics committee of The First Affiliated Hospital of the University of Science and Technology of China (2021-RE -037) and followed the guidelines of the "Declaration of Helsinki." As this was a retrospective study and the data were analyzed anonymously, informed consent of the subjects was not required.

\section{Data Collection}

The clinical data of the 137 patients were collected and analyzed retrospectively, including demographic data, disease-related data, laboratory examination results within 24 hours after AKI, drug and surgical treatment, and complications. Variables recorded included gender; age; blood pressure and mean arterial pressure within 24 hours after $\mathrm{AKI}$; the use of cardio-pulmonary resuscitation (CPR), mechanical ventilation (MV) or surgical treatment during hospitalization; whether the complications of shock (including hemorrhagic shock, septic shock, cardiogenic shock), sepsis or organ failure (including liver failure, heart failure, respiratory failure) occurred, use of vasoactive drugs, hemodialysis, or continuous renal replacement therapy (CRRT). Specific analysis indicators are detailed in Table 1. The 137 patients were divided into "survival" (100 cases) and "death" (37 cases) groups based on whether they survived to discharge or not, respectively.

\section{Statistical Methods}

All data were analyzed using $\mathrm{R}$ software ( $\mathrm{R}$ 3.6.1). Continuous data that met assumptions of a normal distribution were summarized using mean \pm standard deviation (SD), and an independent sample $t$-test was used for comparison between groups. The median plus quartiles 1 and 3 (Q1, Q3) were used to summarize continuous data that did not conform to the normal distribution, and the Mann-Whitney test was used to compare between groups. Classification data were expressed as the number of cases, percentage or rate. The Pearson chi-square test or Fisher's exact probability method were used for comparison between groups in category data. The least absolute shrinkage and selection operator (LASSO) regression method was used to identify the relevant variables for in-hospital death in AKI patients, and then a stepwise multivariate logistic regression analysis based on the minimum Akaike information criterion (AIC) was used to identify the independent risk factors of in-hospital death in AKI patients. The nomogram model and calibration curve were plotted using the "rms" package (Regression Modeling Strategies) in R software. ${ }^{9}$ The receiver operating characteristic (ROC) curve of the nomogram model was plotted using the "pROC" package (an open-source package for R and S+ to analyze and compare ROC curves). ${ }^{10}$ The "rmda" package (Risk Model Decision Analysis) ${ }^{11}$ was used to plot the decision curve analysis (DCA) curve of the nomogram model. To prevent overfitting of the nomogram model, and following the prediction model establishment and internal validation 
Table I Univariate Analysis of the Risk of Poor Prognosis in ICU Inpatients with AKI

\begin{tabular}{|c|c|c|c|c|c|}
\hline Variables & $\begin{array}{c}\text { Total } \\
(n=137)\end{array}$ & $\begin{array}{l}\text { Survival Group } \\
\qquad(\mathrm{n}=100)\end{array}$ & $\begin{array}{c}\text { Death Group } \\
\quad(n=37)\end{array}$ & Statistic & $\mathbf{P}$ \\
\hline Age & $64(52,74)$ & $61.5(49.75,70)$ & $73(63,77)$ & $-3.46^{\mathrm{a}}$ & $<0.001$ \\
\hline Systolic blood pressure within 24 hours after Aki & $109(97,140)$ & $\begin{array}{c}123.5(99.75 \\
\mid 46.5)\end{array}$ & $100(90,106)$ & $4.228^{\mathrm{a}}$ & $<0.001$ \\
\hline Diastolic blood pressure within 24 hours after Aki & $67(52,80)$ & $72(57.75,84.25)$ & $55(45,64)$ & $3.618^{\mathrm{a}}$ & $<0.001$ \\
\hline Mean arterial pressure within 24 hours after Aki & $83(66.33,100)$ & $89(71.92,102.42)$ & $67(64,76.33)$ & $4.06^{\mathrm{a}}$ & $<0.001$ \\
\hline $\mathrm{SCr}$ & $\begin{array}{c}336.95(188 \\
518.3)\end{array}$ & $349(186.5,542)$ & $\begin{array}{c}302(190.5 \\
480)\end{array}$ & $0.868^{\mathrm{a}}$ & 0.386 \\
\hline Blood urea nitrogen & $\begin{array}{c}28.56(15.64 \\
37.3)\end{array}$ & $28.63(15.5,36.48)$ & $\begin{array}{c}28.54(20.8 \\
39.1)\end{array}$ & $-0.722^{\mathrm{a}}$ & 0.470 \\
\hline Uric acid & $533(404,672)$ & $\begin{array}{c}534.5(4 I I, \\
666.45)\end{array}$ & $522(371,675)$ & $0.264^{\mathrm{a}}$ & 0.792 \\
\hline Cystatin C & $2.72(1.75,4)$ & $2.71(1.7,4.23)$ & $\begin{array}{c}2.81(1.85 \\
3.37)\end{array}$ & $0.432^{\mathrm{a}}$ & 0.666 \\
\hline Albumin value & $31.54 \pm 6.32$ & $32.53 \pm 5.99$ & $28.84 \pm 6.49$ & $3.137^{b}$ & 0.002 \\
\hline Platelet value & $137(96,183)$ & $\begin{array}{c}\text { I46.5 (107.5 } \\
\text { I9I.25) }\end{array}$ & $108(76,145)$ & $2.943^{\mathrm{a}}$ & 0.003 \\
\hline Hemoglobin value & $94(80,114)$ & $94(80.75,111.5)$ & $91(77,123)$ & $-0.056^{\mathrm{a}}$ & 0.956 \\
\hline Erythrocrit & $\begin{array}{c}0.28(0.24 \\
0.33)\end{array}$ & $0.28(0.24,0.34)$ & $\begin{array}{c}0.27(0.22 \\
0.33)\end{array}$ & $0.55^{\mathrm{a}}$ & 0.582 \\
\hline White blood cell count $\left(* 10^{9}\right)$ & $\begin{array}{c}9.17(6.27 \\
14.67)\end{array}$ & $8.15(5.72,13.88)$ & $\begin{array}{c}12.7(8.93 \\
16.45)\end{array}$ & $-2.412^{\mathrm{a}}$ & 0.016 \\
\hline Percentage of neutrophils (\%) & $\begin{array}{c}59.2(13.12 \\
84)\end{array}$ & $\begin{array}{c}61.45(12.57 \\
80.78)\end{array}$ & $\begin{array}{c}51.9(13.53, \\
88.9)\end{array}$ & $-0.64^{\mathrm{a}}$ & 0.522 \\
\hline Lymphocyte percentage (\%) & $4.3(0.76,15)$ & $5.88(0.84,16.95)$ & $2.4(0.67,6.7)$ & $1.786^{\mathrm{a}}$ & 0.074 \\
\hline Blood potassium & $\begin{array}{c}4.38(3.87 \\
4.92)\end{array}$ & $4.43(3.87,4.92)$ & $4.27(3.75,4.9)$ & $0.565^{\mathrm{a}}$ & 0.572 \\
\hline $\begin{array}{l}\text { Gender, } \mathrm{n}(\%) \\
\text { Female } \\
\text { Male }\end{array}$ & $\begin{array}{l}44(32.12) \\
93(67.88)\end{array}$ & $\begin{array}{l}36(36) \\
64(64)\end{array}$ & $\begin{array}{c}8(21.62) \\
29(78.38)\end{array}$ & $2.56 \mathrm{I}^{\mathrm{c}}$ & 0.110 \\
\hline $\begin{array}{l}\text { Hypertension, n (\%) } \\
\text { No } \\
\text { Yes }\end{array}$ & $\begin{array}{l}65(47.45) \\
72(52.55)\end{array}$ & $\begin{array}{l}51(5 I) \\
49(49)\end{array}$ & $\begin{array}{l}14(37.84) \\
23(62.16)\end{array}$ & $1.876^{\mathrm{c}}$ & 0.171 \\
\hline $\begin{array}{l}\text { Diabetes, n(\%) } \\
\text { No } \\
\text { Yes }\end{array}$ & $\begin{array}{l}99(72.26) \\
38(27.74)\end{array}$ & $\begin{array}{l}73(73) \\
27(27)\end{array}$ & $\begin{array}{l}26(70.27) \\
\text { II (29.73) }\end{array}$ & $0.1^{\mathrm{c}}$ & 0.751 \\
\hline $\begin{array}{l}\text { Stroke, } n(\%) \\
\text { No } \\
\text { Yes }\end{array}$ & $\begin{array}{c}103(75.18) \\
34(24.82)\end{array}$ & $\begin{array}{l}80(80) \\
20(20)\end{array}$ & $\begin{array}{l}23(62.16) \\
14(37.84)\end{array}$ & $4.606^{\mathrm{c}}$ & 0.032 \\
\hline
\end{tabular}


Table I (Continued).

\begin{tabular}{|c|c|c|c|c|c|}
\hline Variables & $\begin{array}{c}\text { Total } \\
(n=137)\end{array}$ & $\begin{array}{c}\text { Survival Group } \\
(\mathrm{n}=100)\end{array}$ & $\begin{array}{c}\text { Death Group } \\
\quad(n=37)\end{array}$ & Statistic & $\mathbf{P}$ \\
\hline Heart disease, $n(\%)$ & & & & $2.053^{c}$ & 0.152 \\
\hline No & $73(53.28)$ & $57(57)$ & $16(43.24)$ & & \\
\hline Yes & $64(46.72)$ & $43(43)$ & $21(56.76)$ & & \\
\hline History of kidney disease, $\mathrm{n}(\%)$ & & & & $4.415^{\mathrm{c}}$ & 0.036 \\
\hline No & $88(64.23)$ & $59(59)$ & $29(78.38)$ & & \\
\hline Yes & $49(35.77)$ & $4 I(4 I)$ & $8(21.62)$ & & \\
\hline Anemia, $\mathrm{n}(\%)$ & & & & $0.47 I^{c}$ & 0.492 \\
\hline No & $90(65.69)$ & $64(64)$ & $26(70.27)$ & & \\
\hline Yes & $47(34.31)$ & $36(36)$ & II (29.73) & & \\
\hline Tumor, n(\%) & & & & - & $0.779^{d}$ \\
\hline No & $119(86.86)$ & $86(86)$ & $33(89.19)$ & & \\
\hline Yes & $18(13.14)$ & $14(14)$ & $4(10.81)$ & & \\
\hline Blood transfusion, $\mathrm{n}(\%)$ & & & & $0.04^{c}$ & 0.842 \\
\hline No & $102(74.45)$ & $74(74)$ & $28(75.68)$ & & \\
\hline Yes & $35(25.55)$ & $26(26)$ & $9(24.32)$ & & \\
\hline Gastrointestinal bleeding, n (\%) & & & & $1.255^{c}$ & 0.263 \\
\hline No & $112(81.75)$ & $84(84)$ & $28(75.68)$ & & \\
\hline Yes & $25(18.25)$ & $16(16)$ & $9(24.32)$ & & \\
\hline Intestinal obstruction, n (\%) & & & & - & $0.469^{d}$ \\
\hline No & I 35 (98.54) & $99(99)$ & $36(97.3)$ & & \\
\hline Yes & $2(1.46)$ & $\mathrm{I}(\mathrm{I})$ & I (2.7) & & \\
\hline Multiple injuries, $n(\%)$ & & & & - & $1.000^{\mathrm{d}}$ \\
\hline No & $133(97.08)$ & $97(97)$ & $36(97.3)$ & & \\
\hline Yes & $4(2.92)$ & $3(3)$ & I (2.7) & & \\
\hline Hepatobiliary and pancreatic diseases, $n(\%)$ & & & & $2.005^{\mathrm{c}}$ & 0.157 \\
\hline No & $117(85.4)$ & $88(88)$ & $29(78.38)$ & & \\
\hline Yes & $20(14.6)$ & $12(12)$ & $8(21.62)$ & & \\
\hline Pulmonary infection, $n(\%)$ & & & & $2.338^{\mathrm{c}}$ & 0.126 \\
\hline No & $59(43.07)$ & $47(47)$ & $12(32.43)$ & & \\
\hline Yes & $78(56.93)$ & $53(53)$ & $25(67.57)$ & & \\
\hline CPR, n (\%) & & & & - & $<0.001^{d}$ \\
\hline No & $126(91.97)$ & $97(97)$ & $29(78.38)$ & & \\
\hline Yes & II (8.03) & $3(3)$ & $8(21.62)$ & & \\
\hline MV, n (\%) & & & & $23.568^{c}$ & $<0.001$ \\
\hline No & $89(64.96)$ & $77(77)$ & $12(32.43)$ & & \\
\hline Yes & $48(35.04)$ & $23(23)$ & $25(67.57)$ & & \\
\hline Surgery, n(\%) & & & & $3.846^{\mathrm{c}}$ & 0.050 \\
\hline No & II 16 (84.67) & $8 I(8 I)$ & 35 (94.59) & & \\
\hline Yes & $21(15.33)$ & $19(19)$ & $2(5.4 I)$ & & \\
\hline Hemorrhagic shock, n (\%) & & & & - & $0.563^{\mathrm{d}}$ \\
\hline No & I34 (97.8I) & $97(97)$ & $37(100)$ & & \\
\hline Yes & $3(2.19)$ & $3(3)$ & $0(0)$ & & \\
\hline
\end{tabular}

(Continued) 
Table I (Continued).

\begin{tabular}{|c|c|c|c|c|c|}
\hline Variables & $\begin{array}{c}\text { Total } \\
(n=137)\end{array}$ & $\begin{array}{c}\text { Survival Group } \\
(n=100)\end{array}$ & $\begin{array}{c}\text { Death Group } \\
\quad(n=37)\end{array}$ & Statistic & $\mathbf{P}$ \\
\hline Septic shock, n(\%) & & & & $9.223^{c}$ & 0.002 \\
\hline No & $100(72.99)$ & $80(80)$ & $20(54.05)$ & & \\
\hline Yes & $37(27.01)$ & $20(20)$ & $17(45.95)$ & & \\
\hline Cardiogenic shock, n(\%) & & & & - & $0.387^{\mathrm{d}}$ \\
\hline No & 130 (94.89) & $96(96)$ & 34 (91.89) & & \\
\hline Yes & $7(5.11)$ & $4(4)$ & $3(8.11)$ & & \\
\hline Sepsis, $n(\%)$ & & & & - & $1.000^{\mathrm{d}}$ \\
\hline No & $127(92.7)$ & $93(93)$ & 34 (91.89) & & \\
\hline Yes & $10(7.3)$ & $7(7)$ & $3(8.11)$ & & \\
\hline Vasoactive drugs, $n(\%)$ & & & & $34.684^{\mathrm{c}}$ & $<0.001$ \\
\hline No & $104(75.91)$ & $89(89)$ & $15(40.54)$ & & \\
\hline Yes & $33(24.09)$ & II (II) & $22(59.46)$ & & \\
\hline Liver failure, $n(\%)$ & & & & $1.625^{c}$ & 0.202 \\
\hline No & $113(82.48)$ & $85(85)$ & $28(75.68)$ & & \\
\hline Yes & $24(17.52)$ & $15(15)$ & $9(24.32)$ & & \\
\hline Heart failure, n(\%) & & & & $0.083^{c}$ & 0.773 \\
\hline No & $106(77.37)$ & $78(78)$ & $28(75.68)$ & & \\
\hline Yes & $31(22.63)$ & $22(22)$ & $9(24.32)$ & & \\
\hline Respiratory failure, $n(\%)$ & & & & $6.742^{\mathrm{c}}$ & 0.009 \\
\hline No & $87(63.5)$ & $70(70)$ & $17(45.95)$ & & \\
\hline Yes & $50(36.5)$ & $30(30)$ & $20(54.05)$ & & \\
\hline Hemodialysis, n(\%) & & & & $0.083^{c}$ & 0.773 \\
\hline No & $106(77.37)$ & $78(78)$ & $28(75.68)$ & & \\
\hline Yes & $31(22.63)$ & $22(22)$ & $9(24.32)$ & & \\
\hline Start CRRT, n(\%) & & & & $9.08^{c}$ & 0.003 \\
\hline No & $66(48.18)$ & $56(56)$ & $10(27.03)$ & & \\
\hline Yes & $7 \mid(5 I .82)$ & $44(44)$ & 27 (72.97) & & \\
\hline
\end{tabular}

Notes: ${ }^{a}$ mann-Whitney test, ${ }^{\mathrm{b}}$ Independent sample $t$-test, ${ }^{\mathrm{c}}$ Pearson chi-square test, ${ }^{\mathrm{d}}$ Fisher exact probability method.

standard in the "Transparent reporting of a multivariable prediction model for individual prognosis or diagnosis" (TRIPOD) statement, ${ }^{12}$ the bootstrap method (resampling 1000 times) was used for internal validation of the nomogram model. A $P$-value $<0.05$ was considered statistically significant.

\section{Results}

\section{Comparison of Clinical Data Between Groups}

The in-hospital mortality of AKI patients in the ICU was $27.01 \%(37 / 137)$. The median age of subjects was 64 $(52,74)$ years-old, and the sample included $93(67.88 \%)$ males and $44(32.12 \%)$ females. In the 137 subjects, 72
(52.55\%) patients had hypertension, 38 (27.74\%) had diabetes and 34 (24.82) had suffered a stroke. Differences between the death group and survival group were statistically significant $(\mathrm{P}<0.05)$, for the following variables: age, systolic blood pressure within $24 \mathrm{~h}$ of AKI onset (hereafter, "systolic BP"), diastolic blood pressure within $24 \mathrm{~h}$ of AKI onset, mean arterial pressure within $24 \mathrm{~h}$ of AKI onset, albumin value, platelet value, white blood cell count, occurrence of a stroke, history of kidney disease, whether CPR was performed during hospitalization (hereafter, "CPR"), whether MV was performed during hospitalization (hereafter, "MV"), whether surgery was performed during hospitalization (hereafter, "surgery"), presence of infectious shock, application of vasoactive 
drugs (hereafter, "vasoactive drugs"), presence of respiratory failure and whether CRRT was initiated. See Table 1 for details.

\section{Variables Related to In-Hospital Death in ICU Patients with AKI}

Given the many variables identified as statistically significant by univariate analysis (Table 1) and the substantially fewer patients in the death group, the "glmnet" package ${ }^{13}$ was used for LASSO regression to further explore the 16 identified variables and hone in on those variables characteristic of in-hospital death in AKI patients. When selecting the optimal lambda parameters, 10 times cross validation was used, the lambda value at the time of minimum cross validation error was taken as the optimal value of the model (Figure 1) and the number of variables with a non-zero regression coefficient counted. LASSO regression showed that the lambda value when the crossvalidation error was minimized was 0.0263 . This corresponded to a model in which nine variables had a non-zero regression coefficient, including age, systolic BP, albumin value, platelet value, a history of kidney disease, CPR, $\mathrm{MV}$, surgery, and vasoactive drugs. See Figure 1A and $\mathrm{B}$ and Table 2 for details.

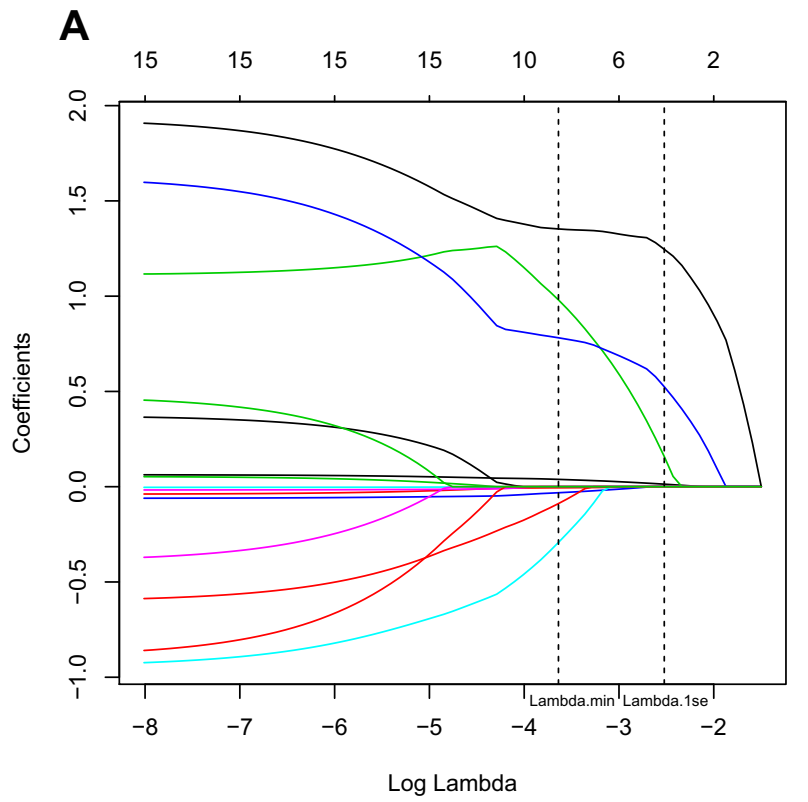

\section{Multivariate Regression Analysis of In-Hospital Mortality Risk of AKI Patients in ICU}

The clinical outcome of 137 patients with AKI was used as the dependent variable, and the nine predictor variables identified by the LASSO regression used as the independent variables in a multivariate logistic regression model. The variable selection method used was a stepwise regression based on minimum Akaike Information Criterion (AIC) ${ }^{14}$ Multivariate logistic regression analysis based on minimum AIC showed that age, albumin value, CPR, $\mathrm{MV}$, surgery, and vasoactive drugs were independent risk factors for in-hospital death of AKI patients (Figure 2).

\section{Development of the Nomogram Model for Predicting In-Hospital Mortality Risk of ICU Inpatients with AKI}

Although only the regression coefficients of age, MV and vasoactive drugs were statistically significant $(\mathrm{P}<0.05)$, considering the clinical practicability of minimal AIC screening variables, we included all six variables identified by multivariate regression analysis based on the minimal AIC when constructing the nomogram model. The nomogram model for predicting the in-hospital mortality risk of

B

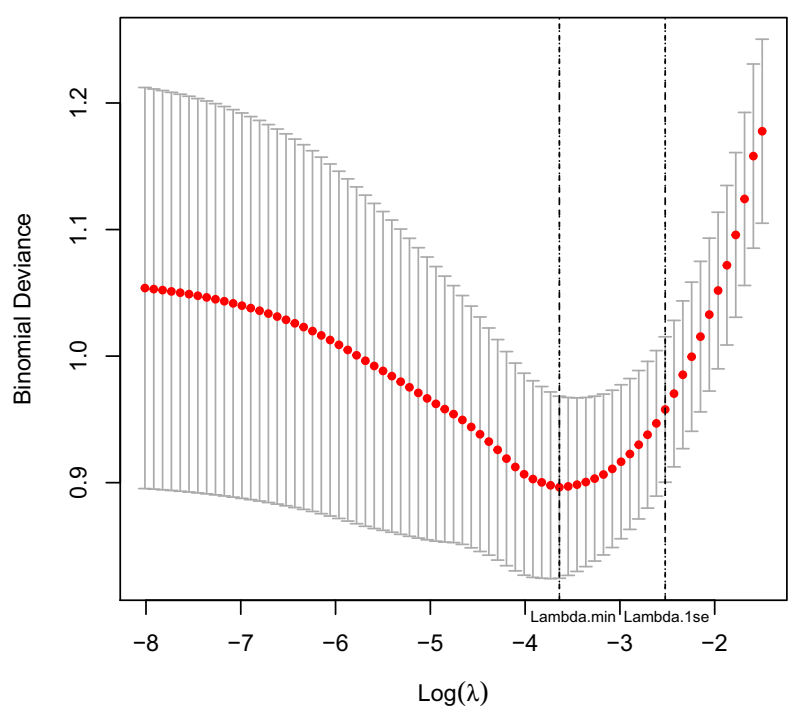

Figure I Coefficient penalty chart of 16 risk factors related to hospital death. (A) Lambda value and the coefficient of 16 variables corresponding to the trend chart. (B) The relationship between lambda value and the LASSO regression coefficient. On both plots, the intersection of the left-most vertical dashed line and the log(lambda) axis represents the $\log ($ lambda) value at lambda minimum and the intersection of the right-most vertical dashed line and the log(lambda) axis represents the log(lambda) value at I SE lambda.

Abbreviation: LASSO regression, Least absolute shrinkage and selection operator regression. 
Table 2 Results of the LASSO Regression

\begin{tabular}{|l|c|}
\hline Variables & Coefficient of the LASSO Regression \\
\hline Constant & -1.319 \\
Age & 0.616 \\
Vasoactive drugs & 0.579 \\
MV & 0.373 \\
CPR & 0.266 \\
Albumin value & -0.200 \\
Systolic blood pressure within 24 hours after AKI & -0.172 \\
Surgery & -0.105 \\
Platelet value & -0.052 \\
History of kidney disease & -0.042 \\
\hline
\end{tabular}

Abbreviations: LASSO regression, Least absolute shrinkage and selection operator regression; CPR, cardio-pulmonary resuscitation; MV, mechanical ventilation; AKI, acute kidney injury.

ICU hospitalized AKI patients was plotted with the "rms" package (Figure 3). Timing for the collection of predictors in the nomogram model was as follows. Age was collected from the admission assessment sheet when the patient was admitted to the ICU. Albumin value was tested and collected within 24 hours of AKI diagnosis. After AKI was diagnosed, the status of the predictors regarding use of CPR, MV, surgery, or vasoactive drugs was updated at the time of use.

\section{Analysis of Clinical Applicability and Prediction Efficiency of the Nomogram Model}

The DCA curve of the nomogram model was plotted using the "rmda" package (Figure 4). According to the DCA curve, when the prediction probability threshold was between 0.01 and 0.75 , the nomogram model had the highest level of clinical net benefit and was better than the "all intervention" curve and "no intervention" curve, suggesting that the nomogram model had good clinical applicability. The Harrell's C-index of the nomogram prediction model for internal data was 0.891 (95\% CI: 0.837-0.945). For internal validation, higher values of C-index may simply indicate model overfitting. To prevent overfitting, the nomogram model was verified internally using the bootstrap method. ${ }^{15}$ The area under the ROC curve (AUC) was 0.886 (95\% CI: 0.823-0936) after using the bootstrap method for internal validation, indicating that the nomogram model has good discrimination ability. See Figures 5A and B for details. The Hosmer-Lemeshow goodness of fit test

\begin{tabular}{lccc}
\hline Variables & $\mathrm{OR}(95 \% \mathrm{Cl})$ & P value \\
\hline \multicolumn{1}{c}{ Age } & $1.06(1.02-1.10)$ & 0.004 \\
Albumin value & $0.94(0.86-1.02)$ & 0.133 \\
CPR(Reference=No) & $4.93(0.95-33.97)$ & 0.072 \\
MV(Reference=No) & $3.23(1.12-9.38)$ & 0.029 \\
Surgery(Reference=No) & $0.29(0.03-1.53)$ & & 0.187 \\
$\begin{array}{l}\text { Vasoactive drugs } \\
\text { (Reference=No) }\end{array}$ & $5.93(1.94-19.35)$ & -10 & 0.002 \\
\hline
\end{tabular}

Figure 2 The multivariate regression of in-hospital mortality risk of $A K I$ patients in ICU. The $x$-axis of the second figure in Figure 2 represents the null line, ie, OR $=1$, indicating no statistical association between the studied factor and the outcome. When the error line for the studied factor intersects this line, it suggests that $P>0.05$ and the $95 \% \mathrm{Cl}$ for OR includes I.

Abbreviations: CPR, cardio-pulmonary resuscitation; MV, mechanical ventilation. 
Points

Age

Albumin value

CPR

MV

Surgery

Vasoactive drugs
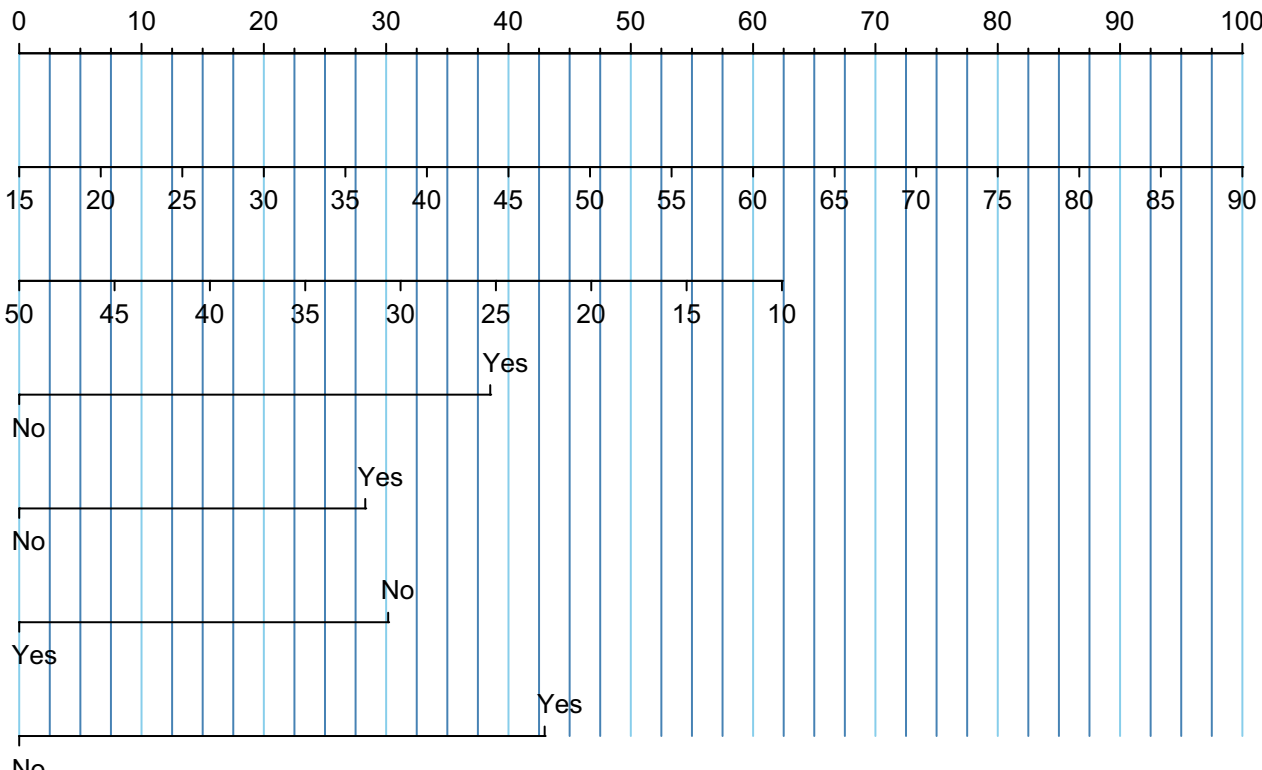

Total Points

$\begin{array}{llllllllllllllll}0 & 20 & 40 & 60 & 80 & 100 & 120 & 140 & 160 & 180 & 200 & 220 & 240 & 260 & 280\end{array}$

In-hospital mortality risk of ICU hospitalized AKI patients

\begin{tabular}{lllll}
\hline 0.1 & 0.3 & 0.5 & 0.7 & 0.9
\end{tabular}

Figure 3 Nomogram model for predicting in-hospital mortality risk in ICU patients with AKI. A vertical line upwards from the specific value of each of the six predictors on the horizontal axis to the horizontal axis of "Points" gives the score. The scores of the six indicators are then summed to obtain the total score. From the position of the total score on the horizontal axis of "Total Points", a vertical line is drawn downward to obtain the probability value on "Risk of poor prognosis in ICU patients with AKI." This is the probability of death for AKI patient in ICU.

Abbreviations: CPR, cardio-pulmonary resuscitation; MV, mechanical ventilation; ICU, intensive care unit; AKI, acute kidney injury.

showed that the prediction deviation between the risk prediction value of the nomogram and the actual frequency of death in ICU patients with AKI was not statistically significant $\left(\chi^{2}=\right.$ 5.660, $\mathrm{P}=0.685$ ). The calibration curve showed that the deviation correction curve of the nomogram model was consistent with the ideal curve, indicating that the nomogram model has good calibration and prediction consistency. See Figure 6A and B for details.

\section{Discussion}

In the last 3 years, a number of reports on the prognosis of patients with AKI in the ICU in China have suggested that in-hospital mortality of AKI patients in the ICU can reach $13.58 \%$ to $60.92 \%{ }^{16-18}$ our data were consistent with this, with an in-hospital mortality rate for AKI patients of $27.01 \%$. The in-hospital mortality of AKI patients in the ICU in China appears to be relatively high. Identifying risk factors for in-hospital mortality of AKI patients in the ICU is thus an important practical step to allow medical staff to identify patients with high risk of in-hospital mortality, implement early warning and prevention strategies, improve the prognosis of patients, and reduce the risk of death.

The results of this study are similar to previous studies $^{19-21}$ that found age, hypoproteinemia, and whether surgery was performed during hospitalization are predictors of in-hospital death in AKI patients. In addition, this study found that use of MV, CPR and vasoactive drugs during hospitalization were closely related to the risk of in-hospital death in ICU patients with AKI. One reason that MV is closely related to mortality risk may be that it is a commonly used life support method in the ICU, through which the life of a critically ill patients can be extended. ${ }^{22}$ However, in recent years, studies have shown that patients under MV for a long time become dependent on the ventilator, leading to difficulties in ventilator withdrawal. Of patients with long-term MV, 25-40\% have difficulty with ventilator withdrawal, and $30-40 \%$ of patients may die from ventilator withdrawal. ${ }^{23-26}$ Vasoactive drugs are commonly used in the treatment of critically ill patients. These drugs need accurate treatment targets, close monitoring for changes in vital signs, titration to the minimum 


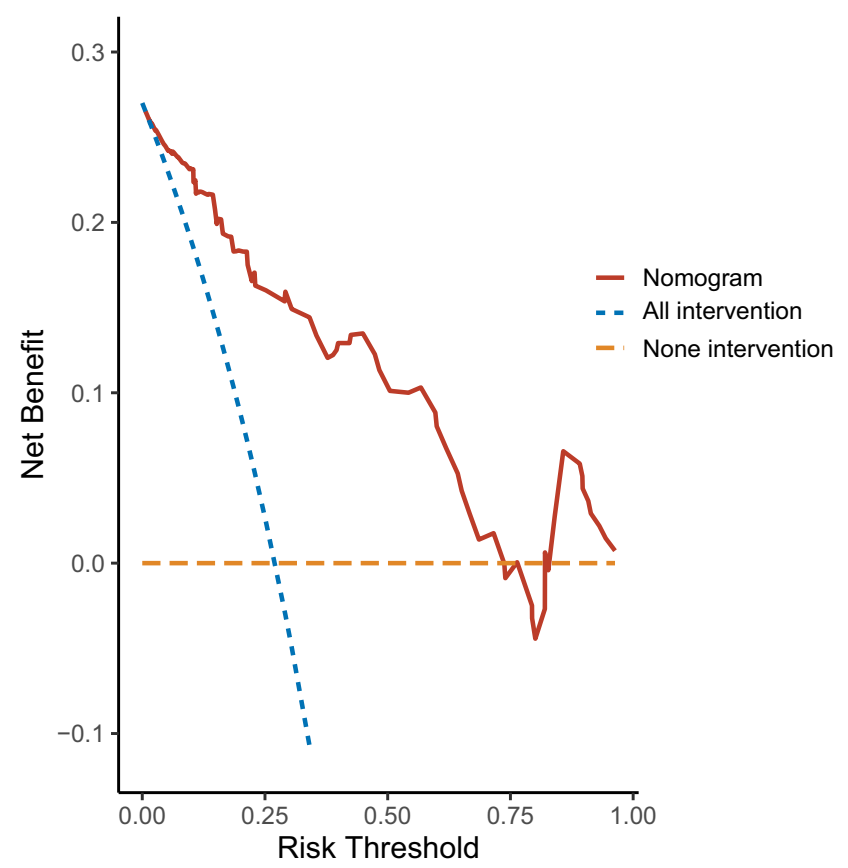

Figure 4 Clinical decision analysis curve of nomogram model. The $x$-axis, the threshold probability, indicates the predicted probability of the occurrence of an event in the sample, based on the model. This is defined as positive for intervention and treatment at this time. The corresponding $y$-axis indicates the net benefit obtained. For the DCA curve of the nomogram model (red-brown solid line), the horizontal dashed line (No intervention, Orange line) indicates the net benefit of not taking the intervention at any threshold probability, and the diagonal dashed line (All interventions, blue line) indicates the net benefit of always taking the intervention.

effective dose and the drug must be stopped as soon as possible, or the hospitalization time of patients will be prolonged, and the risk of adverse prognostic events such

\section{A}

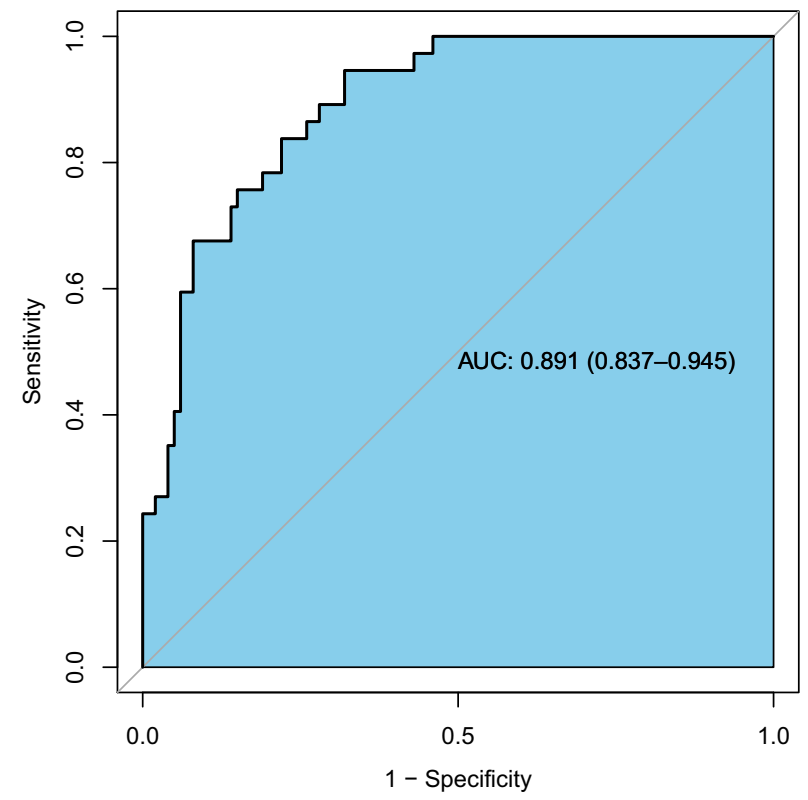

as myasthenia, hypotension, shock and death is increased. ${ }^{27}$ This is consistent with our finding that the proportion of vasoactive drugs used in the death group was significantly higher than that in the survival group for this study. With the continuous improvement and popularization of CPR, the resuscitation rate from cardiac arrest has been correspondingly improved. However, few patients survive and leave hospital after successful resuscitation of cardiac arrest, and the mortality rate of patients after successful CPR is still high, ${ }^{28}$ which was consistent with the results of this study.

A nomogram is a type of visual graph used to predict the probability of a clinical event that intuitively displays the statistical model and accurately quantifies risk. ${ }^{29}$ The nomogram can be used for predicting the risk of AKI related to cardiac surgery, ${ }^{30}$ in septic patients after admission, ${ }^{31}$ and after nephrotic surgery. ${ }^{32}$ This study constructed the first nomogram model to predict the risk of inhospital death in patients with AKI in China based on the risk factors of in-hospital death in the ICU for patients with AKI. Harrell's C-index, AUC of the ROC curve after internal validation, the Hosmer-Lemeshow goodness of fit test and the DCA curve were used to evaluate the predictive efficiency and clinical applicability of the nomogram model. Harrell's C-index indicated the nomogram model has good discrimination ability. The Hosmer-Lemeshow goodness of fit test showed the prediction value of the

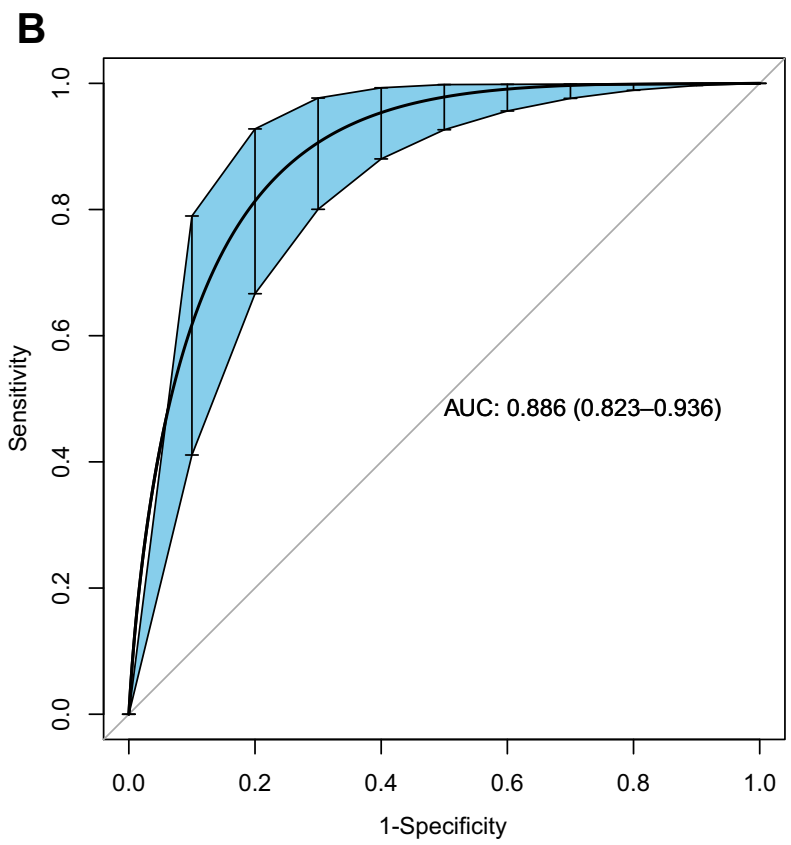

Figure 5 ROC curve of nomogram model. (A) ROC curve before internal validation. (B) ROC curve after internal validation. In both plots the $y$-axis is I minus the sensitivity of the nomogram and the $x$-axis is the specificity of the nomogram.

Abbreviations: ROC, receiver operating characteristic; AUC, area under curve. 
A

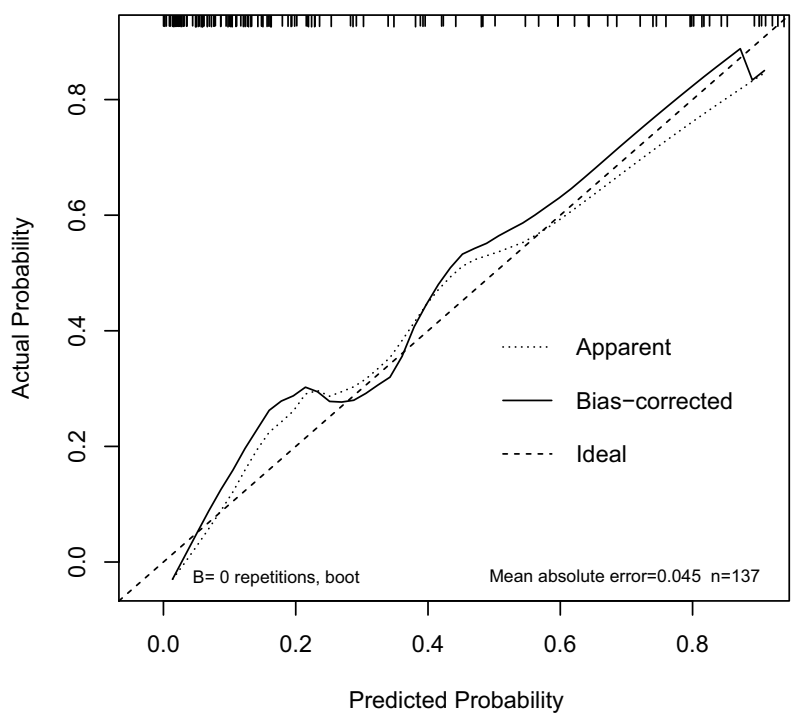

B

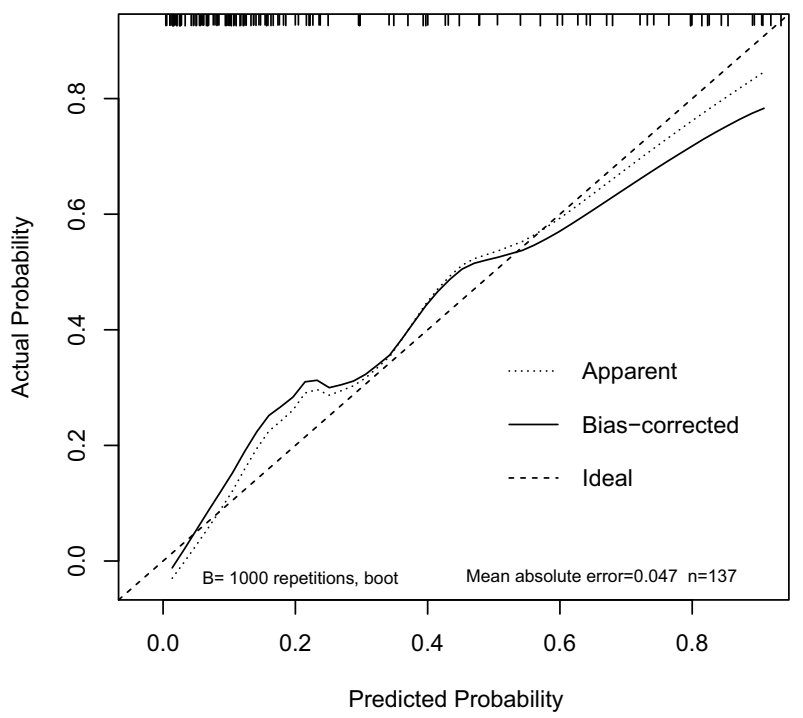

Figure 6 Calibration curve of nomograph model. (A) Calibration curve before internal verification; (B) Calibration curve after internal verification. In both plots, the y-axis indicates the actual probability of patient mortality and the x-axis indicates predicted probability of the patient's mortality in ICU. The 45-degree black dotted line represents the ideal prediction, the solid black line surrounding the 45-degree black dotted line represents the bias-corrected prediction and the black dotted line surrounding the 45degree black dotted line represents the apparent prediction.

nomogram matches the actual frequency of the death in patients with AKI in the ICU. The calibration curve showed that the nomogram model has good calibration and prediction consistency. The DCA curve suggested that the nomogram model had good clinical applicability. Thus, the nomogram model appears to have good prediction efficiency and clinical applicability. It can be used to predict the risk of in-hospital death of ICU hospitalized AKI patients, assist medical staff to screen out patients with high risk of in-hospital death, and carry out targeted risk intervention.

\section{Limitations}

The first limitation of this study is that the data are from a single-center retrospective study that includes complete clinical data for only 137 patients with possible retrospective bias. As such, the data are insufficient to achieve the goal of the study, which would require thousands of patients at multiple centers. Carrying out multicenter, large sample size data collection to analyze the clinical application of the nomogram will be the focus of our next study. Second, this study only carried out internal validation. Validation of the nomogram using external populations to assess prediction efficiency will also be the focus of the next study. Third, the nomogram developed in this study only includes six variables (age, albumin level, CPR, MV, surgical treatment and vasoactive drugs) and this may limit further improvement in the prediction efficiency of the nomogram. It is thus also necessary to carry out prospective research on incorporating more risk factors and improving the prediction efficiency of the nomogram. Fourth, as AKI is a time-varying event that can develop and resolve within the course of an ICU stay, it can be better captured in a time-to-event model rather than a logistic regression model. ${ }^{33}$ At the same time, excess fluid balance in AKI may be harmful and some patients may react to fluid challenges. $^{34}$ Differentiating volume-responsive (VR) and volume-unresponsive (VU) AKI, and analyzing the impact of fluid responsiveness on the final outcome of AKI patients may also be important for predicting the risk of poor prognosis in ICU patients with AKI. Therefore, future prospective studies should consider the prediction efficiency of fluid responsiveness for AKI patients.

\section{Conclusion}

In summary, ICU inpatients with AKI have high in-hospital mortality that is affected by age, albumin level within 24 hours of AKI, whether MV was used, whether vasoactive drugs were used, whether surgery was performed, and whether CPR was performed. The nomogram risk prediction model based on these six risk factors appears to have good prediction efficiency and clinical applicability to help medical staff identify AKI patients with high mortality risk as early as possible and to address relevant risk factors to improve the adverse prognosis of AKI patients. 


\section{Ethics Approval}

The protocol of this study was approved by the medical ethics committee of The First Affiliated Hospital of the University of Science and Technology of China (2021-RE-037) and followed the guidelines of the "Declaration of Helsinki." As this study was a retrospective study and the data was analyzed anonymously, informed consent of the subjects was not required.

\section{Funding}

This study was not supported by any funding.

\section{Disclosure}

The authors declare that the manuscript content was composed in the absence of any commercial or financial relationships that could be construed as a potential conflict of interest.

\section{References}

1. Zhang Q, Guo S, Liang X, et al. Risk prediction and key influencing factors of acute kidney injury in hospitalized patients. Chin J Biomed Eng. 2019;38(06):702-710.

2. Lin L, Chen X, Chen J. Analysis of death related factors of hospitalized super elderly patients with acute kidney injury. Chin J Mod Med. 2020;30(1):115-119.

3. Ruiz-Criado J, Ramos-Barron MA, Fernandez-Fresnedo G, et al. Longterm mortality among hospitalized non-ICU patients with acute kidney injury referred to nephrology. Nephron. 2015;131(1):23-33.

4. Pickering JW, Blunt I, Than MP. Acute kidney injury and mortality prognosis in acute coronary syndrome patients: a meta-analysis. Nephrology. 2018;23(3):237-246. doi:10.1111/nep.12984

5. Yang L, Xing G, Wang L, et al. Acute kidney injury in China: a cross-sectional survey. Lancet. 2015;386(10002):1465-1471. doi:10.1016/S0140-6736(15)00344-X

6. Feng Z. Prospective Epidemiological Study on Acute Kidney Injury of Hospitalized Patients in Multi-Center in China [D]. PLA General Hospital (Beijing 301 Hospital) \& Military Medical College of Continuing Education; 2015.

7. Gong F, Yang X. Pay attention to the recovery of kidney - further discussion on the prevention and treatment strategy of acute kidney injury. J Med Postgrad. 2020;33(01):1-6.

8. Meersch M, Schmidt C, Hoffmeier A, et al. Prevention of cardiac surgery-associated AKI by implementing the KDIGO guidelines in high risk patients identified by biomarkers: the PrevAKI randomized controlled trial. Intensive Care Med. 2017;43(11):1551-1561. doi:10.1007/s00134-016-4670-3

9. Harrell Jr, Frank E. rms: Regression modeling strategies. R package version 5, no. 2, 2016.

10. Robin X, Turck N, Hainard A, et al. pROC: an open-source package for $\mathrm{R}$ and $\mathrm{S}+$ to analyze and compare ROC curves. BMC Bioinform. 2011;12:77. doi:10.1186/1471-2105-12-77

11. Brown M. rmda: Risk Model Decision Analysis[J]. R package version 1.6, 2018.

12. N A Z, Ramspek CL, Dekker FW, et al. TRIPOD statement: a preliminary pre-post analysis of reporting and methods of prediction models. BMJ Open. 2020;10(9):e41537.

13. Friedman J, Hastie T, Tibshirani R. Regularization paths for generalized linear models via coordinate descent. J Stat Softw. 2010;33 (1):1-22. doi:10.18637/jss.v033.i01
14. Zhang Z. Variable selection with stepwise and best subset approaches. Ann Transl Med. 2016;4(7):136. doi:10.21037/atm.2016.03.35

15. Ma YN, Zhang LX, Hu YY, et al. Nomogram model for predicting the risk of multidrug-resistant bacteria infection in diabetic foot patients. Infect Drug Resist. 2021;14:627-637. doi:10.2147/IDR. S287852

16. Ke L, Xie J, Hu Y, et al. Application of support vector machine in prediction of death risk of ICU patients with acute kidney injury. J Peking Univ. 2018;50(2):239-244.

17. Tan Y, Feng X. Analysis of prognostic factors of patients with acute kidney injury in ICU. Chin Mod Doctor. 2020;58(7):97-100.

18. Liang J, Li Z, Dong H, et al. Prognostic factors of 458 patients with acute kidney injury in ICU. J Intern Med Crit Care. 2019;25 (3):202-205.

19. Zhu F. Study on Prognostic Factors and Susceptibility Markers of Elderly Patients with Hospital Acquired Acute Kidney Injury [D]. Medical College of Chinese people's Liberation Army; 2019.

20. Cheng $\mathrm{X}, \mathrm{Wu} \mathrm{B}$, Mao $\mathrm{H}$, et al. Influencing factors of short-term prognosis in patients with acute kidney injury. J Kidney Dis Dialysis Kid Transplant. 2016;25(03):238-244.

21. Chandiraseharan VK, Kalimuthu M, Prakash TV, et al. Acute kidney injury is an independent predictor of in-hospital mortality in a general medical ward: a retrospective study from a tertiary care centre in south India. Indian J Med Res. 2020;152(4):386-392. doi:10.4103/ ijmr.IJMR_1685_18

22. Jia S, He $\bar{Z}$, Teng J, et al. Effect of early progressive rehabilitation training on neuromuscular function recovery of severe patients with mechanical ventilation. Prog Mod Biomed. 2017;17(04):660-663.

23. Sansone GR, Frengley JD, Horland A, et al. Effects of reinstitution of prolonged mechanical ventilation on the outcomes of 370 patients in a long-term acute care hospital. J Intensive Care Med. 2018;33 (9):527-535. doi:10.1177/0885066616683669

24. Wang Z, Teng L, Meng X, et al. Analysis of the causes of difficulty in weaning patients with long-term mechanical ventilation in intensive care unit and the influencing factors of death. Adv Mod Biomed. 2019;19(22):4308-4311.

25. Zhang B, Nie Q, Wang W, et al. Application effect of treatment decision support in family members of patients with long-term mechanical ventilation in ICU. Nurs Res. 2018;32 (21):3453-3456.

26. Muzaffar SN, Gurjar M, Baronia AK, et al. Predictors and pattern of weaning and long-term outcome of patients with prolonged mechanical ventilation at an acute intensive care unit in North India. Rev Bras Ter Intensiva. 2017;29(1):23-33. doi:10.5935/0103507X.20170005

27. Zhang S, Sun R, Mao Z, et al. Analysis of death factors of diabetic nephropathy patients in ICU based on mimic - III database. Chin Crit Care Med. 2020;32(9):1085-1090.

28. Li L. Analysis of outcomes and risk factors of 90 patients with cardiopulmonary resuscitation. J Cardio Cerebrovasc Dis Integr Tradit Chin West Med. 2018;16(10):1462-1464.

29. Chen M, Zheng W, Chen H, et al. Establishment and validation of nomogram prediction model for constipation risk in patients with type 2 diabetes. Acta Nurs Sin. 2021;28(02):58-61.

30. Guan C, Li C, Xu L, et al. Risk factors of cardiac surgery-associated acute kidney injury: development and validation of a perioperative predictive nomogram. J Nephrol. 2019;32(6):937-945. doi:10.1007/ s40620-019-00624-z

31. Deng F, Peng M, Li J, et al. Nomogram to predict the risk of septic acute kidney injury in the first $24 \mathrm{~h}$ of admission: an analysis of intensive care unit data. Ren Fail. 2020;42(1):428-436. doi:10.1080/0886022X.2020.1761832

32. $\mathrm{Xu} \mathrm{L,} \mathrm{Li} \mathrm{C,} \mathrm{Zhao} \mathrm{L,} \mathrm{et} \mathrm{al.} \mathrm{Acute} \mathrm{kidney} \mathrm{injury} \mathrm{after} \mathrm{nephrectomy:}$ a new nomogram to predict postoperative renal function. $B M C$ Nephrol. 2020;21(1):181. doi:10.1186/s12882-020-01839-0 
33. Zhang Z, Reinikainen J, Adeleke KA, Pieterse ME, GroothuisOudshoorn CGM. Time-varying covariates and coefficients in cox regression models. Ann Transl Med. 2018;6(7):121. doi:10.21037/ atm.2018.02.12
34. Zhang Z, Ho KM, Hong Y. Machine learning for the prediction of volume responsiveness in patients with oliguric acute kidney injury in critical care. Crit Care. 2019;23(1):112. doi:10.1186/s13054-0192411-z

\section{Publish your work in this journal}

Risk Management and Healthcare Policy is an international, peerreviewed, open access journal focusing on all aspects of public health, policy, and preventative measures to promote good health and improve morbidity and mortality in the population. The journal welcomes submitted papers covering original research, basic science, clinical \& epidemiological studies, reviews and evaluations, guidelines, expert opinion and commentary, case reports and extended reports. The manuscript management system is completely online and includes a very quick and fair peer-review system, which is all easy to use. Visit http://www.dovepress.com/testimonials.php to read real quotes from published authors. 